\title{
Validation of the prediction rules identifying drug-resistant pathogens in community-onset pneumonia
}

This article was published in the following Dove Press journal: Infection and Drug Resistance

\author{
Daisuke Kobayashi, ${ }^{1,2}$ Yuichiro \\ Shindo, ${ }^{2}$ Ryota Ito, ${ }^{2,3}$ Mai \\ Iwaki, 4,5 Junya Okumura, 2,6 \\ Toshihiro Sakakibara, ${ }^{2,7}$ Ikuo \\ Yamaguchi, ${ }^{8}$ Tetsuya Yagi, ${ }^{9}$ \\ Tomohiko Ogasawara, ${ }^{4}$ \\ Yasuteru Sugino, ${ }^{6}$ Hiroyuki \\ Taniguchi, ${ }^{7}$ Hiroshi Saito, ${ }^{10}$ \\ Hideo Saka," Takashi \\ Kawamura,' Yoshinori \\ Hasegawa $^{2}$
}

On behalf of the Central Japan Lung Study Group

'Kyoto University Health Service, Kyoto, Japan; ${ }^{2}$ Department of Respiratory Medicine, Nagoya University Graduate School of Medicine, Nagoya, Japan; ${ }^{3}$ Division of Infectious Diseases, University of Pittsburgh School of Medicine, Pittsburgh, PA, USA; ${ }^{4}$ Department of Respiratory Medicine, Nagoya Daini Red Cross Hospital, Nagoya, Japan; ${ }^{5}$ Department of Respiratory Medicine, Kasugai Municipal Hospital, Kasugai, Japan; ${ }^{6}$ Department of Respiratory Medicine, Toyota Memorial Hospital, Toyota, Japan; ${ }^{7}$ Department of Respiratory Medicine and Allergy, Tosei Genera Hospital, Seto, Japan; ${ }^{8}$ Department of Central Laboratory, Toyohashi Municipal Hospital, Toyohashi, Japan; ' Department of Infectious Diseases, Nagoya University Hospital, Nagoya, Japan; ${ }^{10}$ Department of Respiratory Medicine, Aichi Cancer Center Aichi Hospital, Okazaki, Japan; "Department of Respiratory Medicine. National Hospital Organization, Nagoya Medical Center, Nagoya, Japan

Correspondence: Yuichiro Shindo Department of Respiratory Medicine, Nagoya University Graduate School of Medicine, 65 Tsurumai-cho, Showa-ku, Nagoya 466-8550, Japan

Tel +8I 527442167

Fax +8I 527442176

Email yshindo@med.nagoya-u.ac.jp
Background: Appropriate initial antibiotic treatment and avoiding administration of unnecessary broad-spectrum antibiotics are important for the treatment of pneumonia. To achieve this, assessment of risk for drug-resistant pathogens (DRPs) at diagnosis is essential.

Purpose: The aim of this study was to validate a predictive rule for DRPs that we previously proposed (the community-acquired pneumonia drug-resistant pathogen [CAP-DRP] rule), comparing several other predictive methods.

Patients and methods: A prospective observational study was conducted in hospitalized patients with community-onset pneumonia at four institutions in Japan. Pathogens identified as not susceptible to ceftriaxone, ampicillin-sulbactam, macrolides, and respiratory fluoroquinolones were defined as CAP-DRPs.

Results: CAP-DRPs were identified in $73(10.1 \%)$ of 721 patients analyzed. The CAP-DRP rule differentiated low vs high risk of CAP-DRP at the threshold of $\geq 3$ points or 2 points plus any of methicillin-resistant Staphylococcus aureus specific factors with a sensitivity of 0.45 , specificity of 0.87 , positive predictive value of 0.47 , negative predictive value of 0.87 , and accuracy of 0.79 . Its discrimination performance, area under the receiver operating characteristic curve, was 0.73 (95\% confidence interval $0.66-0.79)$. Specificity of the CAP-DRP rule against CAP-DRPs was the highest among the six predictive rules tested.

Conclusion: The performance of the predictive rules and criteria for CAP-DRPs was limited. However, the CAP-DRP rule yielded high specificity and could specify patients who should be treated with non-broad-spectrum antibiotics, eg, a non-pseudomonal $\beta$-lactam plus a macrolide, more precisely.

Keywords: antibiotic resistance, algorithms, community-acquired pneumonia, healthcareassociated pneumonia

\section{Introduction}

Pneumonia is one of the lethal infectious diseases. ${ }^{1}$ To achieve appropriate initial antibiotic treatment is essential for patients with pneumonia since inappropriate antibiotic treatment results in adverse outcomes. ${ }^{2,3}$ Identifying patients at risk for drug-resistant pathogens (DRPs) is therefore critical.

To classify patients at risk for DRPs, the 2005 American Thoracic Society (ATS) and Infectious Diseases Society of America (IDSA) guidelines for adult pneumonia proposed the concept of health care-associated pneumonia (HCAP). ${ }^{4}$ The HCAP criteria include nursing home residents, prior hospitalization, receiving home infusion therapy or home wound care, and chronic dialysis. ${ }^{4}$ However, previous studies have shown low prevalence of DRPs in patients with HCAP and low predictability of HCAP 
criteria for identifying patients with DRPs. ${ }^{5-9}$ Thus, there has been an increase of unnecessary broad-spectrum antibiotic use. ${ }^{10}$ In fact, our previous study revealed that overuse of broad-spectrum antibiotics significantly increased the risk of death in patients with non-DRPs. ${ }^{3}$ Therefore, it is crucial to identify patients with pneumonia who do not need broadspectrum antibiotics.

Our previous study also suggested that the treatment strategy for both patients with community-acquired pneumonia (CAP) and HCAP could be unified because the risk factors for DRPs were identical between the two categories of pneumonia. ${ }^{11}$ Currently, the antibiotic treatment strategy should be considered combining CAP and HCAP, that is, community-onset pneumonia, based on their common risk factors for DRPs, ${ }^{5,11-13}$ and some investigators have proposed several prediction rules identifying patients with DRPs at the diagnosis of community-onset pneumonia.,11,14-16 In our previous study, we elucidated six risk factors for CAP-DRPs that were not susceptible to antibiotics commonly used for patients with CAP. The risk factors for CAP-DRPs included prior hospitalization, immunosuppression, previous use of antibiotics, use of gastric acid suppressive agents, tube feeding, and nonambulatory status; and specific risk factors for methicillin-resistant Staphylococcus aureus (MRSA) included chronic dialysis, congestive heart failure, and positive MRSA history. ${ }^{11}$ We proposed a prediction rule based on the cumulative number of risk factors (the CAP-DRP rule). ${ }^{11}$ Practically, patients with no or one risk factor would be at a low risk for CAP-DRPs and those with three or more risk factors would be at a high risk. When patients have two risk factors for CAPDRPs and no MRSA-specific risk factor, they could be also classified into the low risk group for CAP-DRPs.

The aim of this study was to validate the prediction rule, ie, CAP-DRP rule, and to compare its predictive performance with that of others. In addition, we considered it important to identify patients at low risk for CAP-DRPs in order to avoid unnecessary use of broad-spectrum antibiotics associated with worse outcomes.

\section{Materials and methods}

\section{Study design and setting}

This was a prospective observational study conducted in four institutions (a 1,000-bed university hospital and three major community hospitals with $>500$ beds) located in central Japan. Patient data were collected from April 1, 2013 to March 31, 2014. The protocol of this study adhered to the Declaration of Helsinki and the Japanese Ethics Guidelines for Epidemiological Studies. Obtaining informed consent of the participants was waived, but the opt-out method was adopted according to the ethics guidelines. Information about the study was disclosed to the target patients through the Internet, brochures, or bulletin boards at the participating institutions to give the candidates the opportunity to decline participation. This study was approved by the ethical committee of Nagoya University (No. 2012-0338) and the respective institutional review boards of the participating institutions; it was registered at University Hospital Medical Information Network in Japan (No. UMIN000009837; http://www.umin.ac.jp/).

\section{Participants and categories of pneumonia}

The study method was almost identical to that of our previous study. ${ }^{11}$ Briefly, all adult patients (age $\geq 20$ years) who newly developed CAP or HCAP in their daily community living and needed inpatient treatment were enrolled in the study and followed up 1 month later.

\section{Data collection and microbiologic evaluation}

During the patient registration period, the study coordinator (DK) monitored patient enrollment in all institutions to decrease missing and contradiction of data. Microbiologic evaluation was performed by a method similar to a previous study. ${ }^{11}$ Briefly, microbiologic laboratories in all four institutions provided possible causative pathogens, which were cultured in a semiquantitative manner from respiratory tract samples (including sputum, tracheobronchial aspirates, and bronchoalveolar lavage fluid), pleural fluid, and blood. Serologic tests were performed to detect antibodies against Mycoplasma pneumoniae and Chlamydophila pneumoniae. Legionella pneumophila serogroup 1 antigen in urine was tested by immunochromatography. Microbiologic test results were independently reviewed by two investigators (DK and IY). Pathogens provided by the study institutions were recultured. Viruses, acid-fast bacilli, fungus, and anaerobes were not re-cultured. Antibiotic susceptibility tests were performed at a central laboratory (SRL, Inc., Tokyo, Japan). ${ }^{11}$ Microdilution was performed according to the guidelines of the Clinical and Laboratory Standards Institute. ${ }^{17}$ When no breakpoints were specified in these guidelines, clinical breakpoints for bacteria provided by the European Society of Clinical Microbiology and Infectious Diseases (version 1.2) were utilized. ${ }^{18}$

\section{Definition of CAP-DRPs}

Combination therapy with non-antipseudomonal $\beta$-lactam antibiotics plus a macrolide or monotherapy with fluoroquinolone 
has been recommended as the initial empirical antibiotic treatment in the international guidelines of CAP. ${ }^{19}$ Therefore, identified pathogens that were not susceptible to all of the following types of antibiotics: non-antipseudomonal $\beta$-lactam antibiotics (ceftriaxone or ampicillin-sulbactam), macrolides (azithromycin or clarithromycin), and fluoroquinolones (moxifloxacin, levofloxacin, or garenoxacin) were defined as CAP-DRPs.

\section{Outcomes}

In this observational study, we defined the drug resistance of identified pathogens (occurrence of CAP-DRPs) as the main microbiologic outcome. The 30-day and in-hospital mortality were also assessed.

\section{Prediction rules for CAP-DRPs}

Definitions of various prediction rules to identify patients with CAP-DRPs including the CAP-DRP rule are shown in Figure 1. ${ }^{5,11,15,20-22}$

\section{Prediction rules for MRSA}

In our previous study, we elucidated risk factors for MRSA that included three MRSA-specific factors (chronic dialysis during the preceding 30 days, congestive heart failure, and positive MRSA history within the previous 90 days) and three common risk factors for all CAP-DRPs including MRSA (prior hospitalization, prior antibiotic use, and use of gastric acid suppressive agents). ${ }^{11}$ The MRSA-specific risk score was determined on the basis of the cumulative number of risk factors for MRSA. Shorr's MRSA score (range, 0-10) was also calculated according to the original article. ${ }^{14}$

\section{Statistical analysis}

Demographic, clinical, and microbiologic characteristics were described. Categorical data were summarized as frequencies in percentage and continuous data as median with interquartile range.

The sensitivity, specificity, positive predictive value (PPV), negative predictive value (NPV), accuracy, and area under the receiver operating characteristic curve (AUROC) of the prediction rules for CAP-DRP prevalence were evaluated. ${ }^{5,11,14,15,20-22}$ Moreover, we calculated the Youden indexes to compare different thresholds of Shindo's CAP-DRP score. ${ }^{23}$ In this procedure, point estimates and their $95 \%$ confidence intervals (CIs) of the respective parameters were calculated. In addition, the predictive performance of the rules was assessed to identify patients with MRSA. ${ }^{11,14}$ Calculation of sensitivity, specificity, PPV, NPV, and accuracy was performed using the R statistical package (version 3.0.2; R Foundation for Statistical Computing, Vienna, Austria). PASW Statistics 20 (IBM Corporation, Armonk, NY, USA) was used for other statistical analyses. All tests were two-tailed and a $P$-value $<0.05$ was considered statistically significant.

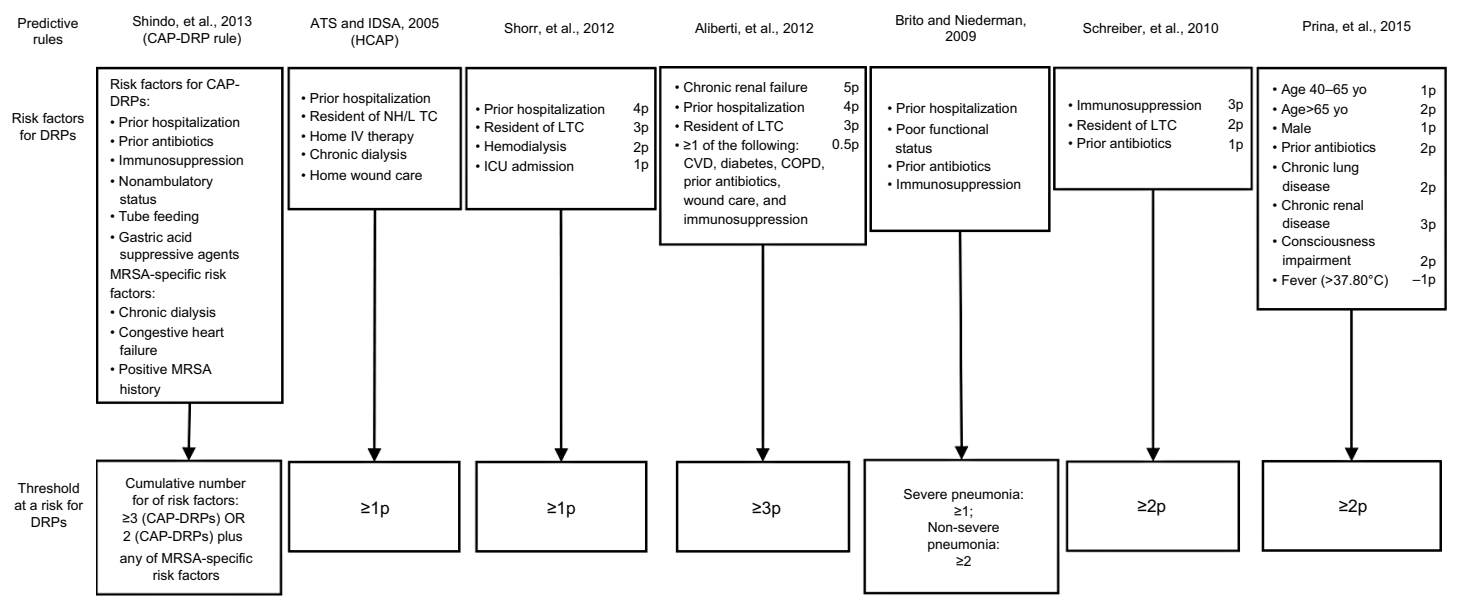

Figure I Definitions of predictive rules for identifying patients with DRPs.

Notes: The definitions of DRPs in each previous report are as follows. Shorr et al: MRSA, Pseudomonas aeruginosa, Acinetobacter baumannii, and ESBL producing organisms; HCAP criteria by ATS and IDSA: P. aeruginosa, ESBL producing Enterobacteriaceae, Acinetobacter sp., and MRSA; Aliberti et al: MRSA, P. aeruginosa resistant to antipseudomonal penicillins, cephalosporins, carbapenems, and quinolones, Stenotrophomonas maltophilia, vancomycin-resistant Enterococcus sp., A. baumannii, ESBL producing Enterobacteriaceae, and other non-fermenting gram-negative bacilli; Brito and Niederman: not defined clearly; Schreiber et al: MRSA, P. aeruginosa, and ESBL producing organisms; Prina et al: MRSA, P. aeruginosa, and ESBL producing Enterobacteriaceae.

Abbreviations: DRPs, drug-resistant pathogens; CAP-DRPs, community-acquired pneumonia drug-resistant pathogens; MRSA, methicillin-resistant Staphylococcus aureus; HCAP, health care-associated pneumonia; NH, nursing home; LTC, long-term care facility; IV, intravenous; CVD, cerebrovascular disease; COPD, chronic obstructive pulmonary disease; ESBL, extended-spectrum $\beta$-lactamase; ICU, intensive care unit; ATS, American Thoracic Society; IDSA, Infectious Diseases Society of America. 


\section{Results}

\section{Demographic and clinical data}

A total of 750 patients with community-onset pneumonia were assessed and 721 patients (480 CAP and 241 HCAP) were enrolled in this study as eligible patients (Figure 2). Among them, 355 patients in whom pathogens were identified and antibiotic susceptibility data were clarified were assessed to validate the prediction rules for CAP-DRPs.
The baseline characteristics of the 721 eligible study patients are described in Table 1. Median age was 77 years and $66.9 \%$ were male. One hundred and twenty-one (16.8\%) patients had a history of prior hospitalization; 265 (36.8\%), prior antibiotic use; 51 (7.1\%), immunosuppression; 146 (20.2\%), nonambulatory status; 25 (3.5\%), tube feeding; and 205 (28.4\%), use of gastric acid suppressive agents.

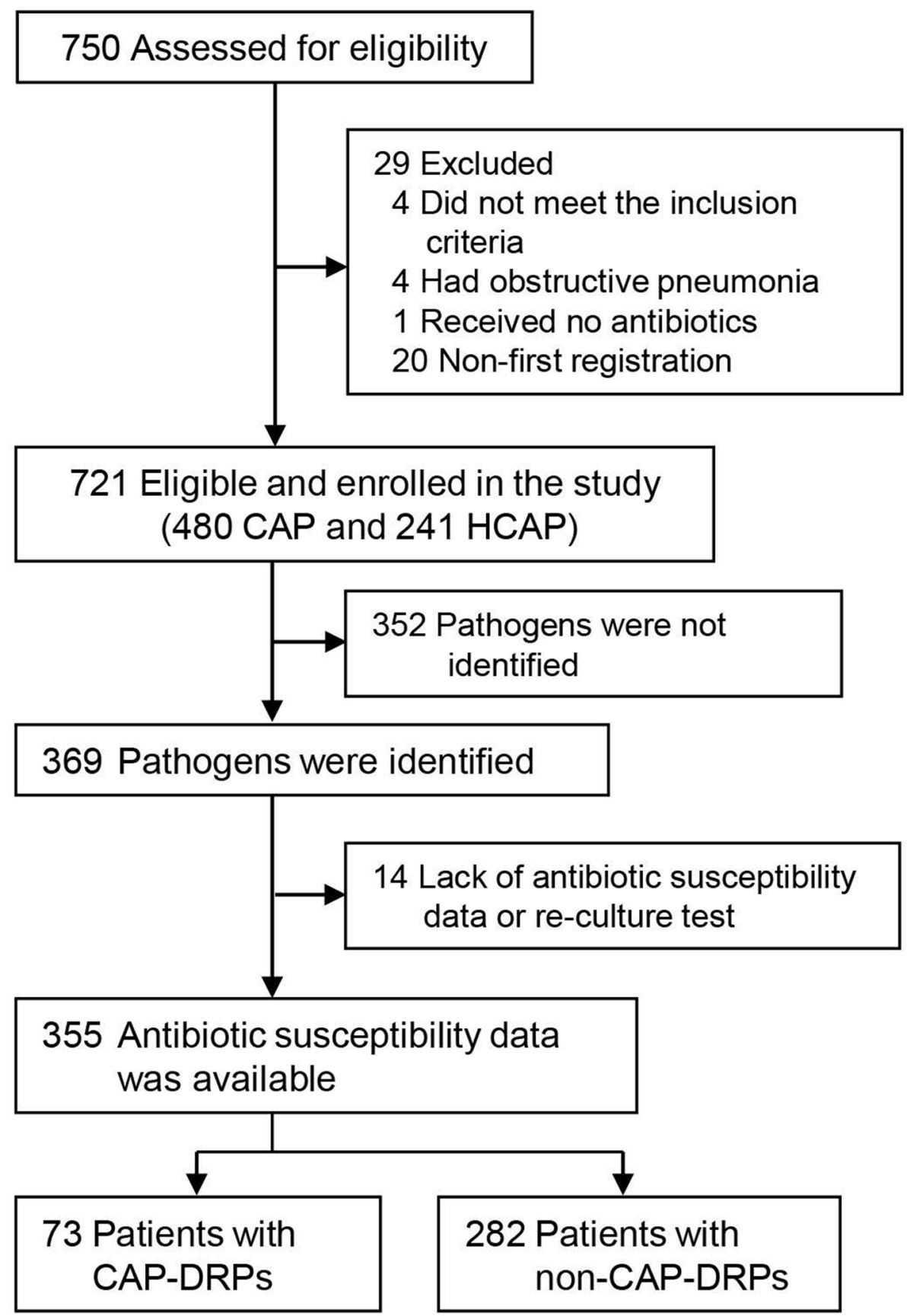

Figure 2 Patient flow.

Abbreviations: CAP, community-acquired pneumonia; HCAP, health care-associated pneumonia; CAP-DRPs, community-acquired pneumonia drug-resistant pathogens. 
Table I Patient characteristics

\begin{tabular}{|c|c|c|}
\hline \multirow[b]{2}{*}{ Age, median (IQR) years } & \multicolumn{2}{|c|}{ Total $(n=721)$} \\
\hline & 77 & $(69-84)$ \\
\hline Men, n (\%) & 482 & $(66.9)$ \\
\hline Health care-associated pneumonia, $\mathrm{n}(\%)$ & 241 & $(33.4)$ \\
\hline $\begin{array}{l}\text { Hospitalization for } \geq 2 \text { days during the preceding } \\
90 \text { days }\end{array}$ & 121 & $(16.8)$ \\
\hline $\begin{array}{l}\text { Resident in a nursing home or extended care } \\
\text { facility }\end{array}$ & 120 & $(16.6)$ \\
\hline $\begin{array}{l}\text { Home intravenous therapy (including antibiotics } \\
\text { and chemotherapy) }\end{array}$ & 37 & $(5.1)$ \\
\hline Chronic dialysis during the preceding 30 days & 12 & $(1.7)$ \\
\hline Home wound care during the preceding 30 days & I & $(0.1)$ \\
\hline Comorbidities, n (\%) & & \\
\hline Chronic lung diseases & 255 & $(35.4)$ \\
\hline Congestive heart failure & 123 & $(17.1)$ \\
\hline Chronic renal diseases & 57 & $(7.9)$ \\
\hline CNS disorders & 117 & $(16.2)$ \\
\hline Diabetes & 126 & $(17.5)$ \\
\hline Immunosuppression ${ }^{\mathrm{a}}$ & 51 & (7.1) \\
\hline Use of antibiotics within the previous & 265 & $(36.8)$ \\
\hline 90 days, $\mathrm{n}(\%)$ & & \\
\hline Nonambulatory status, $\mathrm{n}(\%)$ & 146 & $(20.2)$ \\
\hline Dementia, n (\%) & 112 & $(I 1.5)$ \\
\hline Tube feeding, $\mathrm{n}(\%)$ & 25 & (3.5) \\
\hline Use of gastric suppressive agents, ${ }^{\mathrm{b}} \mathrm{n}(\%)$ & 205 & $(28.4)$ \\
\hline $\begin{array}{l}\text { Positive MRSA history within the previous } 90 \\
\text { days, } n(\%)\end{array}$ & 17 & $(2.4)$ \\
\hline Altered mental status, ${ }^{c} \mathrm{n}(\%)$ & 186 & $(25.8)$ \\
\hline Fever $\left(\mathrm{BT}>37.8^{\circ} \mathrm{C}\right), \mathrm{n}(\%)$ & 307 & $(42.6)$ \\
\hline ICU admission, $\mathrm{n}(\%)$ & 52 & $(7.2)$ \\
\hline PSI class, ${ }^{d}$ n (\%) & & \\
\hline I-III & 277 & $(39.2)$ \\
\hline IV & 270 & $(37.4)$ \\
\hline V & 160 & $(22.2)$ \\
\hline
\end{tabular}

Notes: almmunosuppression included any immunosuppressive diseases, such as congenital or acquired immunodeficiency, hematological diseases, and neutropenia $\left(<1,000 / \mathrm{mm}^{3}\right)$, treatment with immunosuppressive drugs within the previous 30 days, or corticosteroids in daily doses of at least $10 \mathrm{mg} /$ day of a prednisone equivalent for $>2$ weeks. ${ }^{b}$ Gastric suppressive agents included histamine $\mathrm{H}_{2}$-receptor blockers and proton pump inhibitors. ${ }^{c}$ Altered mental status was defined as a Glasgow coma scale $<15$. ${ }^{\text {PSI }}$ was evaluated in 707 patients.

Abbreviations: IQR, interquartile range; CNS, central nervous system; MRSA, methicillin-resistant Staphylococcus aureus; BT, body temperature; ICU, intensive care unit; PSI, Pneumonia Severity Index.

\section{Microbiology and clinical outcomes}

Pathogen distribution and clinical outcomes are shown in Table 2. Pathogens were identified in 369 (51.2\%) patients. Non-CAP-DRPs (eg, Streptococcus pneumoniae, methicillinsensitive S. aureus, Haemophilus influenzae, and antibioticsensitive enteric gram-negative bacilli) were isolated in 296 (41.1\%) of 721 patients with community-onset pneumonia, and CAP-DRPs in 73 (10.1\%). CAP-DRPs included MRSA (51 patients; $7.1 \%)$, Escherichia coli $(12 ; 1.7 \%)$, and Pseudomonas aeruginosa $(9 ; 1.2 \%)$. Among all the study
Table 2 Microbiology and clinical outcomes

\begin{tabular}{|c|l|l|}
\hline & \multicolumn{2}{|l|}{ Total (n=72 I) } \\
\hline Pathogens identified, $\mathrm{n}(\%)$ & 369 & $(5 \mathrm{I} .2)$ \\
CAP-DRPs & 73 & $(10.1)$ \\
MRSA & $5 \mathrm{I}$ & $(7.1)$ \\
Non-MRSA & 27 & $(3.7)$ \\
Escherichia coli & 12 & $(1.7)$ \\
ESBL+ & 8 & $(1.1)$ \\
Pseudomonas aeruginosa & 9 & $(1.2)$ \\
Stenotrophomonas maltophilia & 2 & $(0.3)$ \\
Others & 4 & $(0.6)$ \\
Non-CAP-DRPs & 296 & $(4 I .1)$ \\
Streptococcus pneumoniae & 83 & $(11.5)$ \\
Methicillin-sensitive Staphylococcus & 56 & $(7.7)$ \\
aureus & & \\
Klebsiella pneumoniae & 52 & $(7.2)$ \\
Haemophilus influenzae & 43 & $(6.0)$ \\
Mortality, n (\%) & & \\
30-day & 64 & $(8.9)$ \\
In-hospital & 78 & $(10.8)$ \\
\hline
\end{tabular}

Notes: aldentified pathogens which were not susceptible to $\beta$-lactam antibiotics (ceftriaxone or ampicillin/sulbactam), macrolides (azithromycin or clarithromycin), and fluoroquinolones (moxifloxacin, levofloxacin, or garenoxacin) were defined as CAP-DRPs. '25 isolates of $P$. aeruginosa and 7 of Acinetobacter baumannii were classified as non-CAP-DRPs. ${ }^{\circ}$ Of 81 isolates with antibiotic susceptibility data, 80 were penicillin-susceptible $S$. pneumoniae and one was penicillin intermediate $S$. pneumoniae; and 75 and 74 were resistant to clarithromycin and azithromycin, respectively. Data are presented as $\mathrm{n}(\%)$.

Abbreviations: CAP-DRP, community-acquired pneumonia drug-resistant pathogens; ESBL, extended-spectrum $\beta$-lactamase; MRSA, methicillin-resistant $S$. aureus.

patients, 30-day and in-hospital mortality were $8.9 \%$ and $10.8 \%$, respectively.

\section{Prediction for patients with CAP-DRPs}

First, we examined the validity of the cutoff point of Shindo's CAP-DRP rule. The CAP-DRP rule, $\geq 3$ CAP-DRP risk factors or 2 CAP-DRP risk factors plus any of MRSA-specific risk factors, differentiated patients at high risk from low risk of CAP-DRPs with a sensitivity of 0.45 , specificity of 0.87 , Youden index of 0.32 , PPV of 0.47 , NPV of 0.87 , and accuracy of 0.79 . The simple cutoff to $\geq 2$ and $\geq 3$ CAP-DRP risk factors yielded sensitivity values of 0.59 and 0.33 , specificity of 0.76 and 0.91 , Youden indexes of 0.35 and 0.23 , PPV of 0.37 and 0.46 , NPV of 0.88 and 0.85 , and accuracy of 0.72 and 0.79 , respectively. Considering the Youden index and specificity, $\geq 3$ CAP-DRP risk factors or 2 CAP-DRP risk factors plus any of MRSA-specific risk factors was most preferable.

Second, predictive performance was compared among the six prediction rules and criteria for CAP-DRPs including HCAP ${ }^{4}$ Shorr, ${ }^{15}$ Aliberti, ${ }^{5}$ Brito and Niederman,${ }^{20}$ Schreiber, ${ }^{22}$ 
Prina,${ }^{21}$ and Shindo's CAP-DRP rule (Table 3 ). ${ }^{11}$ When comparing original cutoff scores or criteria that are shown in Figure 1, sensitivity of the CAP-DRP rule (0.45) was lower than those of the other prediction rules, among which that of Aliberti's rule was the highest (0.79). However, specificity of the CAP-DRP rule (0.87) was the highest followed by Brito and Niederman's criteria (0.86). This trend of specificity was almost the same while comparing the prediction rules with changing of cutoff scores so as to have similar levels of sensitivity of the CAP-DRP rule (Table 3 ). In addition, PPV of the CAP-DRP rule was also the highest. NPVs of all tested rules and criteria were around 0.90 .

The CAP-DRP rule was predictive of CAP-DRPs with an AUROC of 0.73 (95\% CI: 0.66-0.79) (Figure 3), which was almost identical to those of Shorr's rule (AUROC: 0.73 [95\% CI: 0.66-0.88]) and Aliberti's rule (AUROC: 0.71 [95\% CI: $0.65-0.77])$, and somewhat larger than that of Schreiber's rule (AUROC: 0.67 [95\% CI: 0.60-0.77]).

\section{Prediction for non-MRSA CAP-DRPs and MRSA}

In our previous study, we proposed the threshold number of risk factors for CAP-DRPs to be three to identify patients with non-MRSA CAP-DRPs. In this validation study, the CAP-DRP rule registered a sensitivity of 0.29 , specificity of 0.87 , PPV of 0.15 , NPV of 0.94 , and accuracy of 0.83 (Table 4). This rule was less sensitive but more specific to non-CAP-DRPs than other rules.

Table 3 Comparison of predictive rules or criteria for CAP-DRPs

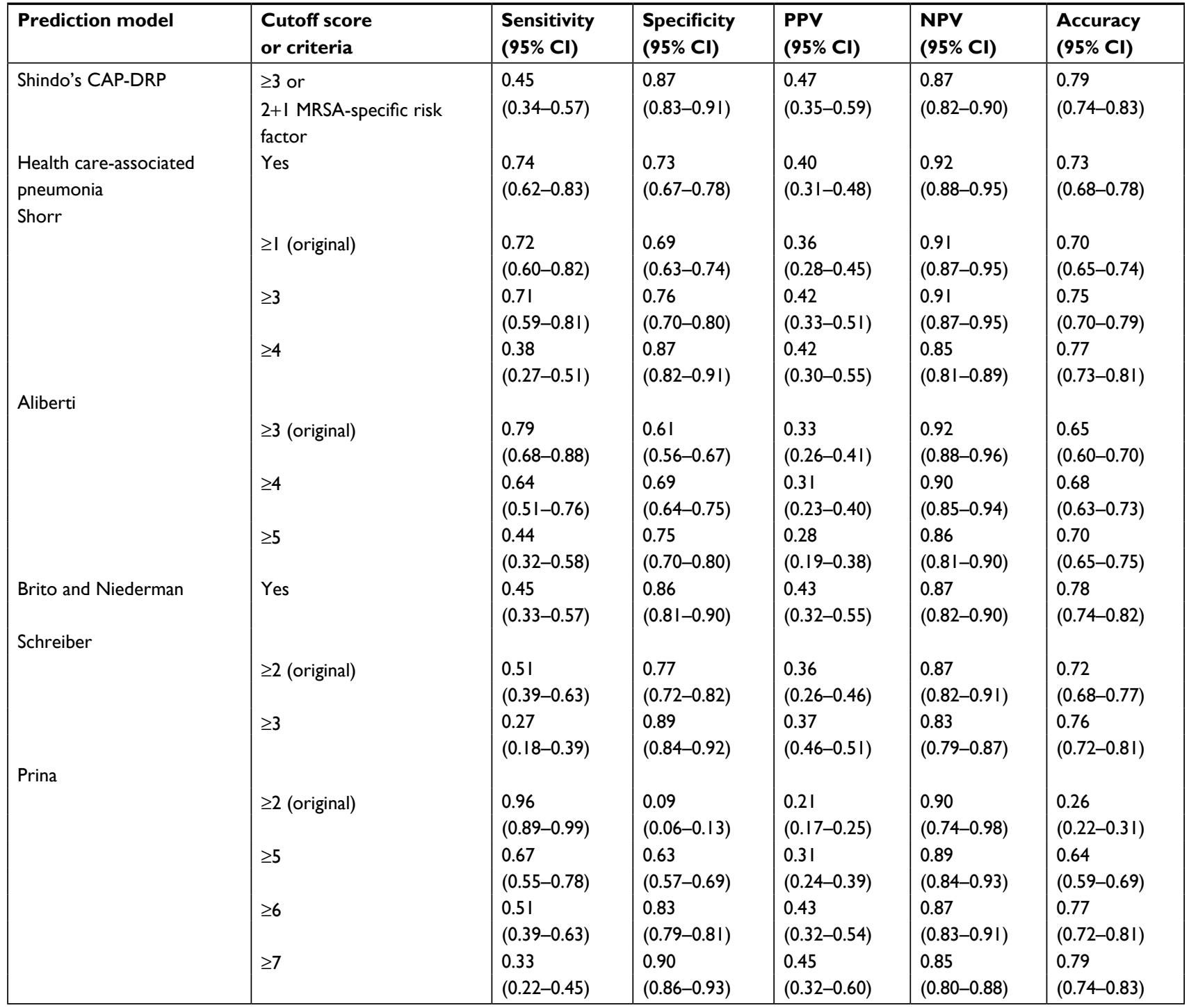

Abbreviations: PPV, positive predictive value; NPV, negative predictive value; $\mathrm{Cl}$, confidence interval; CAP-DRP, community-acquired pneumonia drug-resistant pathogen; MRSA, methicillin-resistant Staphylococcus aureus. 


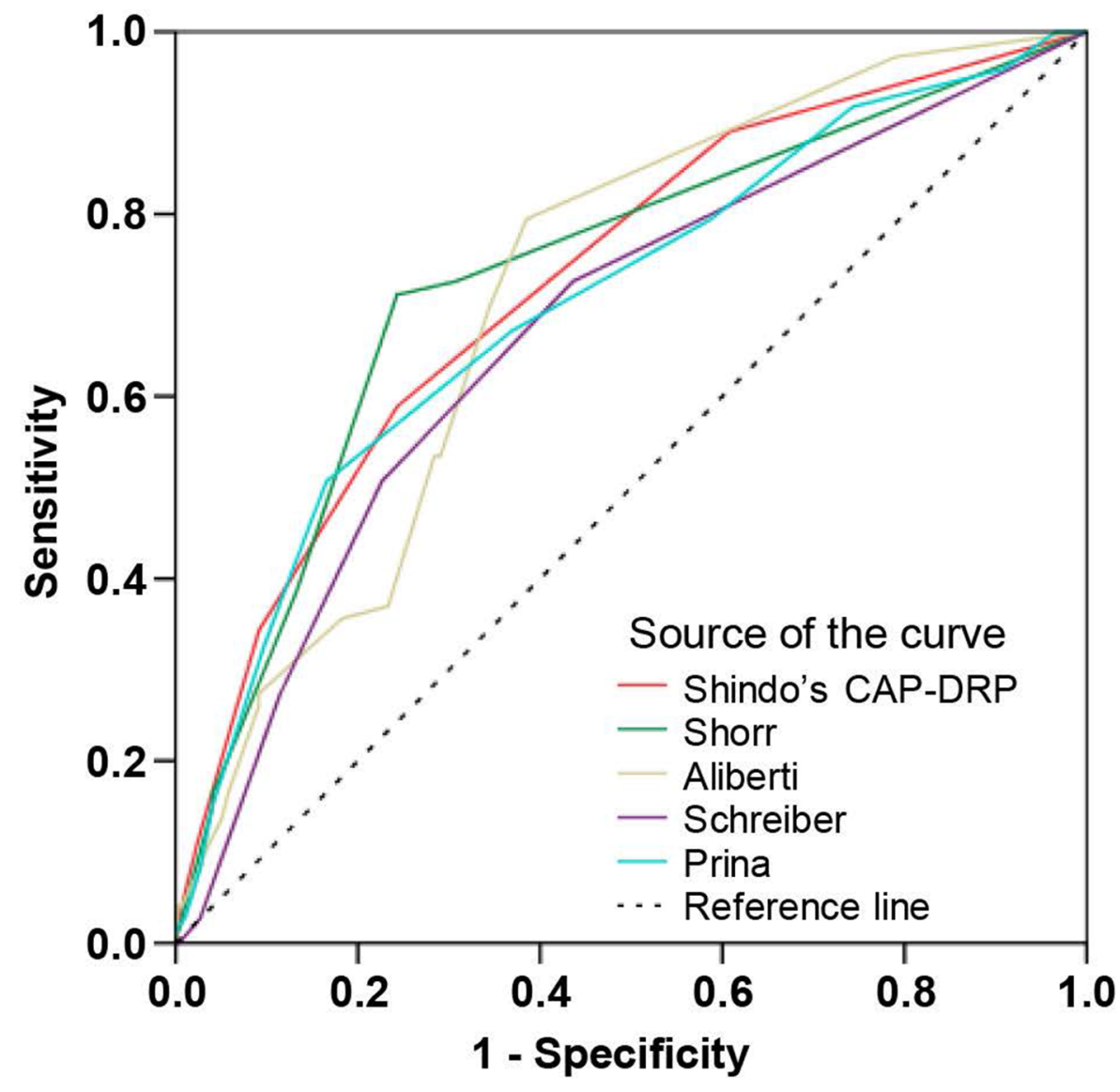

\begin{tabular}{lcc}
\hline Test result variables & Area & $95 \% \mathrm{Cl}$ \\
\hline Shindo's CAP-DRP & 0.73 & $0.66-0.79$ \\
Shorr & 0.73 & $0.66-0.80$ \\
Aliberti & 0.71 & $0.65-0.77$ \\
Schreiber & 0.67 & $0.60-0.74$ \\
Prina & 0.70 & $0.63-0.77$ \\
\hline
\end{tabular}

Figure 3 Receiver operating characteristic curves for predicting CAP drug resistance.

Note: The definitions of each predictive rule are described in Figure I.

Abbreviations: $\mathrm{Cl}$, confidence interval; CAP, community-acquired pneumonia; CAP-DRPs, community-acquired pneumonia drug-resistant pathogens.

The predictive performance of the CAP-DRP rule varying the threshold of MRSA-specific risk factors was also compared to Shorr's MRSA score (Table 4). The CAP-DRP prediction rule of $\geq 3$ CAP-DRP risk factors or that of 2 points plus any of MRSA-specific risk factors differentiated patients with MRSA and non-MRSA with a sensitivity of 0.53 , specificity of 0.86 , PPV of 0.38 , NPV of 0.92 , and accuracy of 0.82 . Furthermore, we also assessed the performance of our MRSA-specific risk estimation by changing the threshold number to 3 and 4 . When the threshold of 4 was adopted, sensitivity and specificity were 0.22 and 0.99 , respectively, with PPV of 0.73 and NPV of 0.89 . Accuracy (0.88) was similar to that of the CAP-DRP rule. Compared to Shorr's MRSA score, the CAP-DRP rule and the MRSA-specific risk factors indicated lower sensitivity and higher specificity. 
Table 4 Predictive rules or criteria for non-MRSA CAP-DRPs and MRSA

\begin{tabular}{|c|c|c|c|c|c|c|}
\hline Prediction model & $\begin{array}{l}\text { Cutoff score or } \\
\text { criteria }\end{array}$ & $\begin{array}{l}\text { Sensitivity } \\
(95 \% \mathrm{Cl})\end{array}$ & $\begin{array}{l}\text { Specificity } \\
(95 \% \mathrm{Cl})\end{array}$ & $\begin{array}{l}\text { PPV } \\
(95 \% \mathrm{Cl})\end{array}$ & $\begin{array}{l}\text { NPV } \\
(95 \% \mathrm{Cl})\end{array}$ & $\begin{array}{l}\text { Accuracy } \\
(95 \% \mathrm{Cl})\end{array}$ \\
\hline \multicolumn{7}{|c|}{ Predictive rules or criteria for non-MRSA CAP-DRPs } \\
\hline Shindo's CAP-DRP & $\geq 3$ & $0.29(0.13-0.49)$ & $0.87(0.83-0.9 \mid)$ & $0.15(0.07-0.28)$ & $0.94(0.90-0.96)$ & $0.83(0.78-0.86)$ \\
\hline $\begin{array}{l}\text { Health care-associated } \\
\text { pneumonia }\end{array}$ & Yes & $0.7 I(0.5 I-0.87)$ & $0.67(0.61-0.72)$ & $0.15(0.09-0.22)$ & $0.97(0.93-0.99)$ & $0.67(0.62-0.72)$ \\
\hline Shorr & $\geq 1$ & $0.64(0.44-0.81)$ & $0.63(0.58-0.68)$ & $0.13(0.08-0.19)$ & $0.96(0.92-0.98)$ & $0.63(0.58-0.68)$ \\
\hline Aliberti & $\geq 3$ & $0.68(0.48-0.84)$ & $0.56(0.50-0.61)$ & $0.11(0.07-0.17)$ & $0.96(0.92-0.98)$ & $0.57(0.5 \mathrm{I}-0.6 \mathrm{I})$ \\
\hline Brito and Niederman & Yes & $0.40(0.22-0.59)$ & $0.81(0.77-0.86)$ & $0.15(0.08-0.25)$ & $0.94(0.91-0.97)$ & $0.78(0.74-0.82)$ \\
\hline Schreiber & $\geq 2$ & $0.54(0.34-0.73)$ & $0.74(0.69-0.79)$ & $0.14(0.08-0.23)$ & $0.95(0.92-0.97)$ & $0.72(0.68-0.77)$ \\
\hline Prina & $\geq 2$ & $1.0(0.82-1.00)$ & $0.09(0.06-0.12)$ & $0.08(0.06-0.12)$ & $1.0(0.83-1.00)$ & $0.16(0.12-0.20)$ \\
\hline \multicolumn{7}{|c|}{ Predictive rules or criteria for MRSA } \\
\hline Shindo's CAP-DRP & $\begin{array}{l}\geq 3 \text { or } 2+I \text { MRSA- } \\
\text { specific factor }\end{array}$ & $0.53(0.39-0.67)$ & $0.86(0.82-0.90)$ & $0.38(0.27-0.50)$ & $0.92(0.88-0.95)$ & $0.82(0.77-0.85)$ \\
\hline $\begin{array}{l}\text { Shindo's MRSA-specific } \\
\text { score }\end{array}$ & $\geq 3$ & $0.4 \mathrm{I}(0.28-0.56)$ & $0.92(0.88-0.95)$ & $0.45(0.30-0.60)$ & $0.91(0.87-0.94)$ & $0.85(0.8 \mathrm{I}-0.88)$ \\
\hline $\begin{array}{l}\text { Shindo's MRSA-specific } \\
\text { score }\end{array}$ & $\geq 4$ & $0.22(0.11-0.35)$ & $0.99(0.97-1.00)$ & $0.73(0.45-0.92)$ & $0.89(0.85-0.92)$ & $0.88(0.84-0.91)$ \\
\hline Shorr's MRSA score & $\geq 2$ & $0.84(0.71-0.93)$ & $0.55(0.49-0.6 \mathrm{I})$ & $0.23(0.17-0.30)$ & $0.96(0.92-0.98)$ & $0.59(0.54-0.64)$ \\
\hline
\end{tabular}

Abbreviations: PPV, positive predictive value; NPV, negative predictive value; Cl, confidence interval; CAP-DRP, community-acquired pneumonia drug-resistant pathogen; MRSA, methicillin-resistant Staphylococcus aureus.

\section{Discussion}

In this multicenter, prospective, observational study, we evaluated the predictive rules for CAP-DRP prevalence in patients with community-onset pneumonia. The CAP-DRP rule demonstrated high specificity in all the predictive rules tested and may reduce the use of unnecessary broad-spectrum antibiotics.

The definition of DRPs is important to discuss the predictive methods. MRSA, extended-spectrum $\beta$-lactamaseproducing Enterobacteriaceae, $P$. aeruginosa, and other non-fermenting gram-negative rods including Acinetobacter baumannii are often defined as potential DRPs. ${ }^{24,25}$ However, some isolates of $P$. aeruginosa and A. baumannii may be susceptible to third-generation cephalosporin and fluoroquinolones. ${ }^{26-28}$ In fact, the definition based on antibiotic susceptibility test results avoided overdiagnosis of DRPs with a reduction of $6.7 \%{ }^{29}$ Therefore, we defined CAP-DRPs as being not susceptible to antibiotics commonly used for patients with CAP (non-pseudomonal $\beta$-lactams, macrolides, and respiratory fluoroquinolones) with emphasis on drug susceptibilities. ${ }^{11,29}$ Our previous study revealed that the risk factors for CAP-DRPs were identical between both patients with CAP and HCAP. ${ }^{11}$ The 2016 IDSA/ATS guidelines for hospital-acquired and ventilator-associated pneumonia state that patients with HCAP should be treated in the same way as CAP. ${ }^{12}$ Thus, a unified treatment strategy for both CAP and HCAP (community-onset pneumonia) should be established, and one of the most important points is to predict patients with CAP-DRPs at diagnosis. ${ }^{11,13,30}$ However, validation studies on predictive rules for DRPs are limited. ${ }^{31,32}$ Therefore, we evaluated the validity of several predictive rules for CAP-DRPs including our proposed rule (CAP-DRP rule) in patients with community-onset pneumonia.

Among the predictive rules tested in this cohort, the CAP-DRP rule differentiated patients at low risk from high risk of CAP-DRPs with high specificity $(0.87$ [95\% CI: 0.83-0.91]). That is, the CAP-DRP rule may identify patients who do not require empirical broad-spectrum antibiotics. Our previous study revealed that antipseudomonal antibiotic use significantly increased the risk of death within 30 days after diagnosis in patients with non-CAP-DRPs such as $S$. pneumoniae and $H$. influenzae. ${ }^{3}$ Therefore, through the avoidance of broad-spectrum antibiotic overuse, patients with no or one risk factor for CAP-DRPs and those with two risk factors for CAP-DRPs plus no MRSA-specific risk factors might improve their outcomes. ${ }^{33}$ However, the AUROC of the CAP-DRP rule was 0.73 , indicating moderate discriminability. ${ }^{34}$ This performance was quite similar to that of Shorr's and Aliberti's rules. Furthermore, even though some tested predictive models had relatively high specificities, their sensitivities tended to be low (Table 3). Therefore, predictive methods may not be suitable for discriminating patients at high risk for CAP-DRPs. For clinical application, predictive methods including the CAP-DRP rule should be refined to achieve higher predictive performance by adding some factors, eg, positive CAP-DRP history, and/or by combining 
with rapid diagnostic test results of antibiotic-resistant genes so that physicians can identify patients with CAP-DRPs in order to select appropriate initial antibiotics.

CAP-DRPs can be divided into MRSA and non-MRSA. Although the separation of MRSA from other CAP-DRPs is important for the selection of therapeutic regimens (ie, anti-MRSA or antipseudomonal antibiotics), ${ }^{35}$ many predictive rules have been created without any consideration for this separation. ${ }^{13}$ In this study, we additionally assessed the predictive performance for MRSA and non-MRSA CAPDRPs separately. While the sensitivity of the CAP-DRP rule for non-MRSA CAP-DRPs was lower than other predictive rules, its specificity was higher than others. Therefore, when a patient has two or less risk factors for CAP-DRPs, antipseudomonal antibiotics should be refrained. Regarding prediction of MRSA, we assessed the predictive performance of the MRSA-specific risk score in addition to the CAP-DRP rule. When the cutoff point of $\geq 4$ was adopted, the specificity was highest (0.99) with a PPV of 0.73 and NPV of 0.89 . When patients have an MRSA-specific risk score of $\leq 3$, anti-MRSA antibiotics would be unnecessary; similarly so in cases with no or one risk factor for CAP-DRPs and two risk factors for CAP-DRPs and none of three MRSA-specific risk factors. Thus, to ensure appropriate antibiotic treatment for patients with CAP-DRPs, it would be preferable to make a distinction between MRSA and non-MRSA CAP-DRPs such as $P$. aeruginosa.

There are some variations between countries or regions regarding risk factors for DRPs, proposed predictive rules, and results of validation studies..$^{13,16,29,31,36,37}$ As Webb et al mentioned, risk factors for DRPs are composed of three factors including host (intrinsic) factors, environment (extrinsic) factors, and selective antibiotic pressure, ${ }^{16}$ the latter of which would differ by countries or regions. ${ }^{29}$ Therefore, predictive rules for DRPs should be created based on local data in the respective countries or regions. If a predictive rule created in a different region is applied, it should be locally validated before clinical use.

In this study, we did not include patients without identified pathogens. Labelle et al and Andruska et al demonstrated that patients with culture-negative pneumonia had lower severity of illness and hospital readmission rate compared with culture-positive patients. ${ }^{38,39}$ Therefore, we considered that patients without identified pathogens should ideally not be analyzed in the same manner as those with identified pathogens. Although prediction models for identifying patients with DRPs were developed among only patients with identified pathogens in most previous studies, ${ }^{5,11,15,21,22}$ physicians must determine the initial antibiotics for both patients with identified pathogens and those without. Therefore, researchers in future should discuss whether patients without identified pathogens (about half of all patients with community-onset pneumonia) should be included when developing the prediction model.

\section{Limitations}

This study had some limitations. First, outpatients were not included in this study. Therefore, the results of this study should be applied in only patients admitted to the hospital. Second, the pathogens identified in this study may not have been the cause of pneumonia in some cases because the cultures were performed semiquantitatively rather than quantitatively. Third, the prediction rules tested in this study were developed based on different definitions of DRPs. The difference between this study and the original ones might affect each predictive performance. Fourth, we could not compare with the Drug Resistance in Pneumonia (DRIP) score that was recently published and includes prior DRP history as a risk factor for DRPs. ${ }^{32}$ We will obtain prior non-MRSA CAPDRP history and evaluate the DRIP score in future studies.

\section{Conclusion}

This multicenter prospective study examined the performance of several predictive rules or criteria for CAP-DRPs in patients with community-onset pneumonia. The predictability of the predictive rules is limited. The CAP-DRP rule yielded high specificity among the tested rules, and can reduce the overuse of broad-spectrum antibiotics and identify patients who can be treated by non-broad-spectrum antibiotics such as a non-antipseudomonal $\beta$-lactam plus a macrolide.

\section{Data availability}

The data sets used and analyzed for the current study are available from the corresponding author on request.

\section{Acknowledgments}

We thank the laboratory staff (Mariko Mochizuki, Yoshiko Sugaki, Yusuke Nishida, Sachie Asai, and Nobuya Sakagami), and all health care professionals who participated in the data collection. Funders of the Central Japan Lung Study Group (CJLSG) had no role in the design and conduct of the study; gathering, management, analysis, and interpretation of data; and preparation of the manuscript. This study was supported by JSPS KAKENHI grant number JP17K09610 and by CJLSG, a nonprofit organization supported by unrestricted donations from the following pharmaceutical 
companies: Chugai Pharmaceutical Co, Ltd; Shionogi \& Co, Ltd; Daiichi Sankyo Co, Ltd; Dainippon Sumitomo Pharma Co, Ltd; Janssen Pharmaceutical KK; Eli Lilly Japan KK; Taisho Toyama Pharmaceutical Co, Ltd; Meiji Seika Pharma Co, Ltd; MSD KK; Bayer Holding Ltd; Astellas Pharma Inc; and Nippon Boehringer Ingelheim Co, Ltd.

\section{Author contributions}

DK planned the study concept, collected the study material, prepared the first draft of the manuscript, and takes responsibility for the integrity of the data and the accuracy of the data analysis. YS, TK, and YH contributed to the study design, data interpretation, statistical analyses, and critical revision of the manuscript. All authors contributed toward data analysis, drafting and critically revising the paper and agree to be accountable for all aspects of the work. All authors read and approved the final manuscript.

\section{Disclosure}

All of the following information provide relevant financial activities outside the submitted work.

Dr Shindo reports receiving personal fees from Pfizer Inc, Shionogi \& Co, Ltd, Beckman Coulter, Inc, MSD KK, Dainippon Sumitomo Pharma Co, Ltd, and Taisho Toyama Pharmaceutical Co, Ltd. Dr Yagi reports receiving grants from Taisho Toyama Pharmaceutical Co, Ltd, Shionogi \& Co, Ltd, Pfizer Inc, Daiichi Sankyo Co, Ltd, Meiji Seika Pharma Co, Ltd, Astellas Pharma Inc, Dainippon Sumitomo Pharma Co, Ltd, and MSD KK; and receiving personal fees from Taisho Toyama Pharmaceutical Co, Ltd, Shionogi \& Co, Ltd, Pfizer Inc, Asahi Kasei Pharma Co, Ltd, Daiichi Sankyo Co, Ltd, Meiji Seika Pharma Co, Ltd, Astellas Pharma Inc, Dainippon Sumitomo Pharma Co, Ltd, MSD KK, and Mitsubishi Tanabe Pharma. Dr Ogasawara reports receiving personal fees from Chugai Pharmaceutical Co, Ltd, Japan Boehringer Ingelheim Co, Ltd, Taiho Pharmaceutical Co, Ltd, Bristol-Myers Squibb Inc, Kyowa Hakko Kirin Pharmaceutical Co, Ltd, and KYORIN Pharmaceutical Co, Ltd. Dr Sugino reports receiving personal fees from Astellas Pharma Inc, Ono Pharmaceutical Co, Ltd, Shionogi \& Co, Ltd, Chugai Pharmaceutical Co, Ltd, Abbott Japan Co, Ltd, MSD KK, Sanofi, GlaxoSmithKline plc, Mitsubishi Tanabe Pharma, Pfizer Inc, Novartis Pharma KK, AstraZeneca KK, KYORIN Pharmaceutical Co, Ltd, and Elsevier Japan KK. Dr Taniguchi reports receiving personal fees from Abbott Japan Co, Ltd, Actelion Pharmaceuticals Japan Ltd, Asahi Kasei Pharma Corp, Astellas Pharma Inc, AstraZeneca
KK, Bayer in Japan, Boehringer Ingelheim Co, Ltd, Chugai Pharmaceutical Co, Ltd, Eli Lilly Japan KK, Fukuda Denshi Co, Ltd, GlaxoSmithKline plc, KYORIN Pharmaceutical Co, Ltd, Meiji Seika Pharma Co, Ltd, Novartis Pharma KK, Ono Pharmaceutical Co, Ltd, Pfizer Japan Inc, Philips Respironics GK, Shionogi \& Co, Ltd, TAIHO Pharmaceutical Co, Ltd, Terumo Corporation, and Teijin Pharma Ltd. Dr Saito reports receiving grants from Ono Pharmaceutical Co, Ltd, Taiho Pharmaceutical Co, Ltd, and Merck Serono Co, Ltd; and receiving personal fees from Ono Pharmaceutical Co, Ltd, Pfizer Inc, Astrazeneca KK, and Kyowa Hakko Kirin Co, Ltd. Dr Saka reports receiving grants from Taisho Toyama Pharmaceutical Co, Ltd, Shionogi \& Co, Ltd, Pfizer Inc, Chugai Pharmaceutical Co, Ltd, Dainippon Sumitomo Pharma Co, Ltd, Bristol-Myers Squibb Company, Kyowa Hakko Kirin Co, Ltd, Eisai Co, Ltd, Ono Pharmaceutical Co, Ltd, Boehringer Ingelheim Co, Ltd, MSD KK, Eli Lilly Japan KK, Daiichi Sankyo Co, Ltd, Astrazeneca KK, Novartis Pharma KK, Taiho Pharmaceutical Co, Ltd, Sanofi KK, and Bayer Yakuhin, Ltd. Dr Kawamura reports receiving personal fees from Sanofi KK. Dr Hasegawa reports receiving grants from Chugai Pharmaceutical Co, Ltd, Daiichi Sankyo Co, Ltd, Astellas Pharma Inc, Dainippon Sumitomo Pharma Co, Ltd, and MSD KK; and receiving personal fees from Taisho Toyama Pharmaceutical Co, Ltd, Shionogi \& Co, Ltd, Pfizer Inc, Chugai Pharmaceutical Co, Ltd, Daiichi Sankyo Co, Ltd, Astellas Pharma Inc, Dainippon Sumitomo Pharma Co, Ltd, MSD KK, Meiji Seika Pharma Co, Ltd, and Abbott Japan Co, Ltd. The other authors report no conflicts of interest in this work.

\section{References}

1. World Health Organization. World Health Statistics; 2014. Available from: http:/www.who.int/mediacentre/news/releases/2014/worldhealth-statistics-2014/en/. Accessed October 28, 2016.

2. Shindo Y, Imaizumi K, Hasegawa Y. Health-Care-Associated Pneumonia Is Primarily Due to Aspiration Pneumonia: Response. Chest. 2009;136(6): 1703.

3. Shindo Y, Ito R, Kobayashi D, et al. Risk factors for 30-day mortality in patients with pneumonia who receive appropriate initial antibiotics: an observational cohort study. Lancet Infect Dis. 2015;15(9): $1055-1065$.

4. American Thoracic Society, Infectious Diseases Society of America. Guidelines for the management of adults with hospital-acquired, ventilator-associated, and healthcare-associated pneumonia. Am J Respir Crit Care Med. 2005;171(4):388-416.

5. Aliberti S, di Pasquale M, Zanaboni AM, et al. Stratifying risk factors for multidrug-resistant pathogens in hospitalized patients coming from the community with pneumonia. Clin Infect Dis. 2012;54(4):470-478.

6. Chalmers JD, Rother C, Salih W, Ewig S. Healthcare-associated pneumonia does not accurately identify potentially resistant pathogens: a systematic review and meta-analysis. Clin Infect Dis. 2014;58(3): $330-339$. 
7. Garcia-Vidal C, Viasus D, Roset A, et al. Low incidence of multidrugresistant organisms in patients with healthcare-associated pneumonia requiring hospitalization. Clin Microbiol Infect. 2011;17(11):1659-1665.

8. Grenier C, Pepin J, Nault V, et al. Impact of guideline-consistent therapy on outcome of patients with healthcare-associated and communityacquired pneumonia. J Antimicrob Chemother. 2011;66(7):1617-1624

9. Jeong B-H, Koh W-J, Yoo H, et al. Risk Factors for Acquiring Potentially Drug-Resistant Pathogens in Immunocompetent Patients with Pneumonia Developed Out of Hospital. Respiration. 2014;88(3):190-198.

10. Yu VL. Guidelines for hospital-acquired pneumonia and health-careassociated pneumonia: a vulnerability, a pitfall, and a fatal flaw. Lancet Infect Dis. 2011;11(3):248-252.

11. Shindo Y, Ito R, Kobayashi D, et al. Risk Factors for Drug-Resistant Pathogens in Community-acquired and Healthcare-associated Pneumonia. Am J Respir Crit Care Med. 2013;188(8):985-995.

12. Kalil AC, Metersky ML, Klompas M, et al. Management of Adults With Hospital-acquired and Ventilator-associated Pneumonia: 2016 Clinical Practice Guidelines by the Infectious Diseases Society of America and the American Thoracic Society. Clin Infect Dis. 2016;63(5) e61-e111.

13. Webb BJ, Jones B, Dean NC. Empiric antibiotic selection and risk prediction of drug-resistant pathogens in community-onset pneumonia. Curr Opin Infect Dis. 2016;29(2):167-177.

14. Shorr AF, Myers DE, Huang DB, Nathanson BH, Emons MF, Kollef $\mathrm{MH}$. A risk score for identifying methicillin-resistant Staphylococcus aureus in patients presenting to the hospital with pneumonia. $B M C$ Infect Dis. 2013;13(1):268.

15. Shorr AF, Zilberberg MD, Reichley R, et al. Validation of a clinical score for assessing the risk of resistant pathogens in patients with pneumonia presenting to the emergency department. Clin Infect Dis. 2012;54(2):193-198.

16. Webb BJ, Dascomb K, Stenehjem E, Dean N. Predicting risk of drugresistant organisms in pneumonia: moving beyond the HCAP model Respir Med. 2015;109(1):1-10.

17. Clinical Laboratory Standards Institute (CLSI). Performance Standards for Antimicrobial Susceptibility Testing. CLSI document M100-S20. Wayne, PA: Clinical Laboratory Standards Institute; 2010.

18. European Society of Clinical Microbiology and Infectious Diseases, European Committee on Antimicrobial Susceptibility Testing. Breakpoint table for interpretation of MICs and zone diameters. Available from: http:/www.eucast.org/fileadmin/src/media/PDFs/EUCAST_files/ Disk_test_documents/EUCAST_breakpoints_v1.2_101220.xls. Accessed July 21, 2012.

19. Mandell LA, Wunderink RG, Anzueto A, et al. Infectious Diseases Society of America/American Thoracic Society Consensus Guidelines on the Management of Community-Acquired Pneumonia in Adults. Clin Infect Dis. 2007;44(Suppl 2):S27-S72.

20. Brito V, Niederman MS. Healthcare-associated pneumonia is a heterogeneous disease, and all patients do not need the same broad-spectrum antibiotic therapy as complex nosocomial pneumonia. Curr Opin Infect Dis. 2009;22(3):316-325.

21. Prina E, Ranzani OT, Polverino E, et al. Risk Factors Associated with Potentially Antibiotic-Resistant Pathogens in Community-Acquired Pneumonia. Ann Am Thorac Soc. 2015;12(2):153-160.
22. Schreiber MP, Chan CM, Shorr AF. Resistant pathogens in nonnosocomial pneumonia and respiratory failure: is it time to refine the definition of health-care-associated pneumonia? Chest. 2010;137(6):1283-1288.

23. Fluss R, Faraggi D, Reiser B. Estimation of the Youden Index and its associated cutoff point. Biom J. 2005;47(4):458-472.

24. Park SC, Kang YA, Park BH, et al. Poor prediction of potentially drugresistant pathogens using current criteria of health care-associated pneumonia. Respir Med. 2012;106(9):1311-1319.

25. Shorr AF, Zilberberg MD, Micek ST, Kollef MH. Prediction of infection due to antibiotic-resistant bacteria by select risk factors for health care-associated pneumonia. Arch Intern Med. 2008;168(20):2205-2210.

26. Hui DS, Ip M, Ling T, et al. A multicentre surveillance study on the characteristics, bacterial aetiologies and in vitro antibiotic susceptibilities in patients with acute exacerbations of chronic bronchitis. Respirology. 2011;16(3):532-539.

27. Yamaguchi K, Ohno A, Ishii Y, Tateda K, Iwata M; LevofloxacinSurveillance Group. [In vitro susceptibilities to levofloxacin and various antibacterial agents of 12,866 clinical isolates obtained from 72 centers in 2010]. Jpn J Antibiot. 2012;65(3):181-206. Japanese.

28. Zhanel GG, Adam HJ, Baxter MR, et al. Antimicrobial susceptibility of 22746 pathogens from Canadian hospitals: results of the CANWARD 2007-11 study. J Antimicrob Chemother. 2013;68(Suppl 1):i7-i22.

29. Shindo Y, Hasegawa Y. Regional differences in antibiotic-resistant pathogens in patients with pneumonia: implications for clinicians. Respirology. 2017;22(8):1536-1546.

30. Wunderink RG. Community-acquired Pneumonia versus Healthcareassociated Pneumonia. The Returning Pendulum. Am J Respir Crit Care Med. 2013;188(8):896-898.

31. SelfWH, Wunderink RG, Williams DJ, Barrett TW, Baughman AH, Grijalva CG. Comparison of clinical prediction models for resistant bacteria in community-onset pneumonia. Acad Emerg Med. 2015;22(6):730-740.

32. Webb BJ, Dascomb K, Stenehjem E, et al. Derivation and Multicenter Validation of the Drug Resistance in Pneumonia Clinical Prediction Score. Antimicrob Agents Chemother. 2016;60(5):2652-2663.

33. Okumura J, Shindo Y, Takahashi K, et al. Mortality in patients with community-onset pneumonia at low risk of drug-resistant pathogens: Impact of beta-lactam plus macrolide combination therapy. Respirology. 2018;23(5):526-534.

34. Singanayagam A, Chalmers JD, Hill AT. Severity assessment in community-acquired pneumonia: a review. QJM. 2009;102(6):379-388.

35. Wunderink RG, Waterer GW. Clinical practice. Community-acquired pneumonia. N Engl J Med. 2014;370(6):543-551.

36. Aliberti S, Cilloniz C, Chalmers JD, et al. Multidrug-resistant pathogens in hospitalised patients coming from the community with pneumonia: a European perspective. Thorax. 2013;68(11):997-999.

37. Sibila O, Rodrigo-Troyano A, Shindo Y, Aliberti S, Restrepo MI Multidrug-resistant pathogens in patients with pneumonia coming from the community. Curr Opin Pulm Med. 2016;22(3):219-226.

38. Andruska A, Micek ST, Shindo Y, et al. Pneumonia Pathogen Characterization Is an Independent Determinant of Hospital Readmission. Chest. 2015;148(1):103-111.

39. Labelle AJ, Arnold H, Reichley RM, Micek ST, Kollef MH. A comparison of culture-positive and culture-negative health-care-associated pneumonia. Chest. 2010;137(5):1130-1137.
Infection and Drug Resistance

\section{Publish your work in this journal}

Infection and Drug Resistance is an international, peer-reviewed openaccess journal that focuses on the optimal treatment of infection (bacterial, fungal and viral) and the development and institution of preventive strategies to minimize the development and spread of resistance. The journal is specifically concerned with the epidemiology of antibiotic resistance and the mechanisms of resistance development and diffusion in both hospitals and the community. The manuscript management system is completely online and includes a very quick and fair peerreview system, which is all easy to use. Visit http://www.dovepress.com/ testimonials.php to read real quotes from published authors. 